\title{
The Management of Psoriatic Arthritis in Italy: Organizational Impact Analysis of Optimized Pathways
}

\author{
Umberto Restelli $^{1,2}$, Carlo Selmi ${ }^{3,4}$, Valeria Pacelli ${ }^{1}$, Davide Croce ${ }^{1,2}$, \\ Antonio Costanzo ${ }^{5,6}$ \\ Center for Health Economics, Social and Health Care Management, LIUC - Università Cattaneo, Castellanza (VA), Italy \\ School of Public Health, Faculty of Health Sciences, University of the Witwatersrand, Johannesburg, South Africa \\ . Division of Rheumatology and Clinical Immunology, Humanitas Research Hospital, Rozzano (MI), Italy \\ 4. BIOMETRA Department, University of Milan, Milan, Italy \\ 5. Dermatology Unit, Department of Biomedical Sciences, Humanitas University and Humanitas Clinical and Research Center, \\ Rozzano (MI), Italy \\ 6. Skin Pathology Laboratory, IRCCS Humanitas, Rozzano (MI), Italy
}

\begin{abstract}
BACKGROUND: The management of psoriatic arthritis requires competencies in the fields of both rheumatology and dermatology, and a multidisciplinary approach.

AIM: To propose an effective pathway for the diagnosis, monitoring and treatment of psoriatic arthritis in the Italian context, and to assess its organizational impact on the Regional Health Service of Lombardy Region.

METHODS: The analysis was performed through interviews conducted with two key opinion leaders in the areas of dermatology and rheumatology. The current pathway of patients who present symptoms that might be related to psoriatic arthritis was defined and an optimized pathway was then proposed on the basis of the clinical practice, considering the implementation of a dermatology and rheumatology shared outpatient service. The organizational impact of the optimized pathway was then assessed from both the hospital and that of the Regional Health Service of Lombardy Region perspectives.

RESULTS: The implementation of the service would have a positive impact on patients' experience, improving the quality of the service provided, thanks to the multidisciplinary approach adopted, limiting the patients' resources needed for the diagnosis, reducing the number of visits and time loss. The optimized pathway, therefore, would have a limited impact on the marketing mix, while potentially improving patients satisfaction, increasing the possibility of patients' retention. To successfully implement the dermatological and rheumatologic multidisciplinary service, a precise communication strategy is mandatory.

CONCLUSIONS: The optimized pathway for the diagnosis and management of psoriatic arthritis proposed would have a limited organizational impact at both hospital and Regional Health Service levels, while leading to theoretical benefits in terms of a prompt diagnosis of the pathology.
\end{abstract}

\section{Keywords}

Psoriatic arthritis; Multidisciplinary service; Organizational impact

\section{BACKGROUND}

Psoriatic arthritis is a chronic inflammatory joint disease, associated with psoriasis [1-4], and is "associated with increased mortality from cardiovascular disease" [5]. Two recent systematic reviews investigated its prevalence at an international level and in Italy [6,7]. Scotti and colleagues [6] evaluated epidemiological studies worldwide (referred to the European context, Northern and Southern American context and Asian context) and reported a pooled prevalence of psoriatic arthritis of 133 cases every 100,000 subjects (with a high betweenstudy heterogeneity), with study prevalence rates ranging from 20 cases every 100,000 subjects (in a study on the Swedish context referred to 1961-1963) [8] to 670 cases every 100,000 subjects (in a study on the Norwegian context referred to 2006-2008) [9]. Prignano and colleagues [7] considered the sole Italian context, identifying two studies which investigated the

Corresponding author Umberto Restelli urestelli@gmail.com Received: 19 March 2019 Accepted: 17 June 2019 Published: 2 July 2019 
prevalence of psoriatic arthritis in two sample populations referred to Marche Region (central Italy, among adults with $\geq 18$ years) and Chiavari (a municipality located in Liguria Region, Northern Italy, among adults with $\geq 16$ years). The first study, referred to 2004, assessed a prevalence of $0.42 \%$ [10,11], while the latter, referred to 1991-1992, assessed a prevalence of $0.09 \%$ [12].

The management of psoriatic arthritis requires competencies in the fields of both rheumatology and dermatology, and a multidisciplinary approach. It is therefore important to implement clinical pathways that allow an efficient management of patients, shortening the time to diagnosis. A timely diagnosis of psoriatic arthritis, in fact, might lead to a prompt therapy start, slowing the disease progression.

The aim of the present analysis is to propose an effective pathway for the diagnosis, monitoring and treatment of psoriatic arthritis in the Italian context, and to assess its organizational impact on the Regional Health Service of Lombardy Region.

\section{METHODS}

The analysis was performed through interviews conducted with two key opinion leaders in the areas of dermatology and rheumatology: the director of the Dermatology ward, and the director of the Rheumatology and Clinical Immunology ward of the Clinical Institute Humanitas located in Rozzano, Milan.

The current pathway of patients who present symptoms that might be related to psoriatic arthritis was defined considering the first access to primary care services, specialist visits, health services for the diagnosis of the pathology, outpatients activities for patients' monitoring and treatments. Three typologies of psoriatic arthritis were considered: predominantly dermatological (in which symptoms are mainly related to the dermatological field), predominantly rheumatologic (axial) and predominantly rheumatologic (peripheral).

An optimized pathway was then proposed on the basis of the clinical practice of the aforementioned key opinion leaders, considering the implementation of a dermatology and rheumatology shared outpatient service, to improve patients' experience and the efficiency of resources allocation.

The organizational impact of the optimized pathway was then assessed from both hospital and Regional Health Service of Lombardy Region perspectives through the use of the Health Technology Assessment (HTA) Core Model $^{\circledR}$ developed by the European Network for Health Technology Assessment (EUnetHTA), version 3.0 [13]. Among the 9 domains considered within the model (Health problem and current use of technology; Description and technical characteristics of technology; Safety; Clinical effectiveness; Costs and economic evaluation; Ethical analysis; Organizational aspects; Patients and Social aspects; Legal aspects) [13] the organizational aspect is declined into 5 items: the health delivery process, the structure of health care system, process-related costs, management, and culture. All the issues considered for each item were analyzed and discussed with the key opinion leaders involved, to identify the consequences of the implementation of the optimized pathway.

\begin{tabular}{lcc}
\hline \multirow{2}{*}{ Outpatient activity } & \multicolumn{2}{c}{ Number of annual activities } \\
\cline { 2 - 3 } & $\begin{array}{c}\text { Psoriatic arthritis } \\
\text { predominantly dermatological }\end{array}$ & $\begin{array}{c}\text { Psoriatic arthritis } \\
\text { predominantly rheumatologic }\end{array}$ \\
\hline Specialist visit & 4 & 4 \\
Complete blood test & 4 & 4 \\
Uric acid & 4 & 4 \\
C-reactive protein & 4 & 4 \\
Glucose test & 4 & 4 \\
Transaminases & 4 & 4 \\
X-rays of the hands and wrists & 1 & 1 \\
Magnetic resonance of the hip & $0.25^{2}$ & $0.5^{3}$ \\
Creatinine & 4 & 4 \\
Alanine aminotransferase & 4 & 4 \\
Vitamin D & 1 & 1 \\
\hline
\end{tabular}

Table I. Annual monitoring scheme for psoriatic arthritis

${ }^{1}$ Axial or peripheral

${ }^{2}$ Every 4 years

${ }^{3}$ Every 2 years 


\section{RESULTS}

The two pathways of patients with symptoms that might be related to psoriatic arthritis are composed by a diagnostic phase and a monitoring/treatment phase.

\section{Currently adopted pathway}

The first step of the pathway consists in a general practitioner visit. The patient might then be addressed to a dermatological specialist visit. If the dermatologist detects symptoms that might be related to psoriatic arthritis, a rheumatologic specialist visit is prescribed. If the suspect of psoriatic arthritis is confirmed, the following diagnostic activities are prescribed: sedimentation velocity, rheumatoid factor, cyclic citrullinated peptide antibody, interferon gamma release assay test, hepatitis $\mathrm{B}$ virus antigen, hepatitis $\mathrm{B}$ virus antibodies, hepatitis $\mathrm{C}$ virus antibodies, ultrasound of muscles and tendons (for each painful area). Once the aforementioned

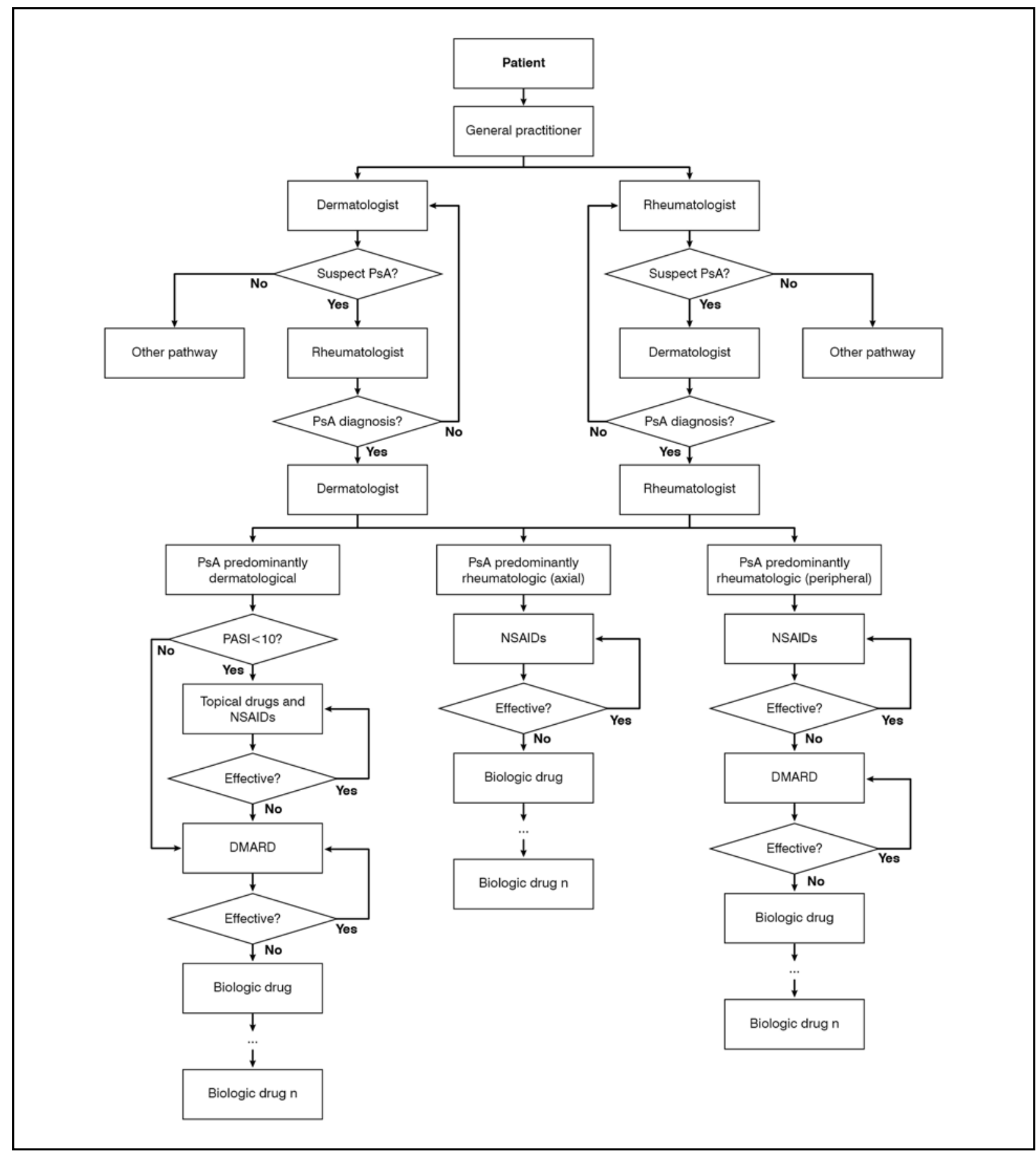

Figure 1. Currently adopted pathway of patients with symptoms potentially related to psoriatic arthritis

DMARD = conventional synthetic Disease Modifying Anti-Rheumatic Drugs; NSAIDs = Non-Steroidal Anti-Inflammatory Drugs; PASI = Psoriasis Area Severity Index; PsA = Psoriatic Arthritis 
tests are performed, a second dermatological specialist visit is performed to confirm or not the psoriatic arthritis diagnosis. This first part of the pathway can be considered in a specular way considering as a first specialist visit a rheumatologic visit instead of a dermatologic one.

Based on the results of the tests, an adequate therapeutic pathway will be set up considering the type of psoriatic arthritis diagnosed: predominantly dermatological, predominantly rheumatologic - axial or predominantly rheumatologic - peripheral. In case the pathology is not diagnosed, the same tests will be repeated after 12 months. A common monitoring scheme will be also implemented, considering the activities reported in Table I.

The schematization of the currently adopted pathway is presented in Figure 1, along with the therapeutic sequences per type of psoriatic arthritis diagnosed to the patient, in terms of treatment typology.

The optimized clinical pathway is characterized by the availability of a dermatology and rheumatology shared outpatient service, that allow the performance of multidisciplinary specialist visits. The patient might approach the service after a primary care visit with his/her general practitioner, who might observe symptoms related to psoriasis and/or

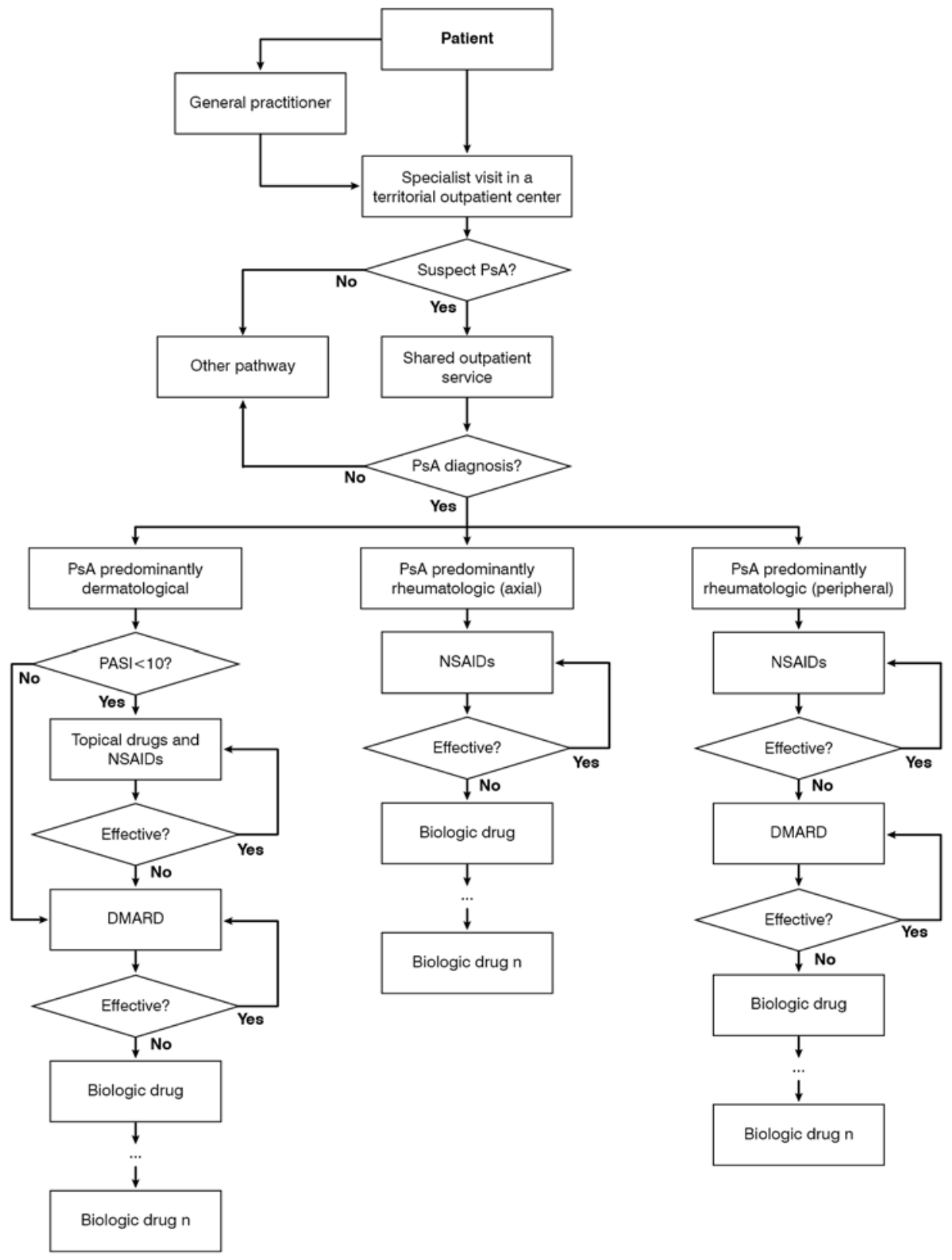

Figure 2. Proposed optimized pathway for patients with symptoms potentially related to psoriatic arthritis

DMARD = conventional synthetic Disease Modifying Anti-Rheumatic Drugs; NSAIDs = Non-Steroidal Anti-Inflammatory Drugs; PASI = Psoriasis Area Severity Index; PsA = Psoriatic Arthritis 
arthritis and can prescribe a dermatological or rheumatologic specialist visit. The specialist visit might be performed at a territorial level by a dermatologist or by a rheumatologist (based on the symptoms observed by the general practitioner) and, if symptoms related to psoriatic arthritis are observed, a specialist visit to the shared outpatient service might be prescribed. Patients might avoid the general practitioner visit, performing a private specialist visit before being addressed to the shared outpatient service. Within this service, a dermatological and rheumatologic multidisciplinary visit will be performed to assess the appropriate diagnostic pathway for psoriatic arthritis (that might consider the activities reported in Table I). Based on the tests results, an adequate therapeutic pathway will be set up, as in the actual pathway, along with monitoring activities. The optimized pathway is represented in Figure 2.

\section{Organizational impact of the optimized pathway}

The results of the organizational impact analysis are reported considering the main topics of the EUnetHTA Core Model $^{\circledR}$ (Version 3.0).

The health delivery process will be partially influenced at a hospital level due to the fact that patients with suspected psoriatic arthritis will be visited within the multidisciplinary outpatient service, requiring the identification of opening days and hours, and of preferential slots to perform diagnostic tests to the patients referring to the service. The processes related to the booking of visits and back office activities would not be affected by the service.

The first step of the process, represented by territorial primary care and specialist visits, would allow only patients with symptoms that might be related to psoriatic arthritis to access the multidisciplinary service. The service would allow an increase in the efficiency of the diagnostic process, avoiding a step (in terms of specialist dermatological or rheumatologic visit), reducing the time needed to confirm the diagnosis. The visit in the multidisciplinary service might be performed by a specialist, with the possibility to request an immediate consultation with a dermatologist or a rheumatologist, optimizing the use of human resources.

No particular involvement for patients and/or caregivers is expected. The optimized pathway might require a reduced number of visits, with the possibility to identify timely symptoms that might be related to psoriatic arthritis. This comprehensive management of the patient might have positive consequences for the hospital, in terms of patient's retention (defined as the ability of the service/hospital to maintain a long term relationship with the patient).

No specific training would be required for the hospital specialists for the implementation of the service. Specific training might be necessary at a primary care level, to instruct general practitioner concerning the symptoms related to psoriatic arthritis, that should be considered to prescribe a specialist visit to diagnose the pathology, and to present them the optimized pathway.

A specific communication activity to inform of the availability of the multidisciplinary service should be addressed to general practitioners and territorial pharmacists. Furthermore, communication to patients' associations should be performed to inform on the availability of the service within the optimized pathway.

No peculiar quality assurance and monitoring systems should be activated beside those already activated within the hospitals that will activate the multidisciplinary service.

From a Regional Health Service perspective, no differences would be assessed considering the health delivery process.

Concerning the structure of the health care system topic, a higher degree of centralization or de-centralization would not influence the implementation of the optimized pathway.

The processes that would allow patients to access the optimized pathway are those described upon, in terms of communication activity with professionals within primary care service. The already existing processes would allow patients to access the multidisciplinary service.

In terms of process-related costs, those related with the implementation of the optimized pathway would depend on each hospital that will activate it, and are related to the physical structure in which the visits will be performed: room, furniture, waiting room. These might be already available within the hospital. Furthermore, considering the fact that the visits of the multidisciplinary service will be programmed, it would be possible to use already available spaces, used to perform dermatological or rheumatologic specialist visits, identifying time slots to be used for these visits.

The optimized pathway would not reduce the diagnostic activity performed in absolute terms, however it would reduce the time to diagnosis. A timely start of psoriatic arthritis 
therapy might lead to benefits to the patients in terms of therapy effectiveness and quality of life (considering the negative effects of psoriatic arthritis on this parameter) $[14,15]$.

The main impact on a hospital budget might be related to the identification and furnishing of the rooms to be dedicated to the multidisciplinary service, however, depending on each hospital activating the service, it is difficult to estimate the potential budget impact, that might be equal to 0 in case of already available spaces. The back office activity related to the booking of the service would be the same already available within each hospital.

If properly perceived at a territorial level and by patients, a potential effect of the optimized pathway might be the increase of specialist visits within the hospital.

From the Regional Health Service perspective, the multidisciplinary service might accelerate the diagnostic phase of the pathway, with positive consequences in terms of prompt therapy start, as described upon, reducing the progress of the pathology with positive consequences on the health services delivered to the patient.

The theoretical increase in the number of diagnosis in the short/medium term, might lead to an increase of health expenditures, due to the increased number of patients starting psoriatic arthritis therapies, being compensated by the improved clinical conditions of patients with a hypothetical reduction of direct medical costs in a long term time horizon, in terms of reduced hospitalizations, outpatient activities and drugs.

Concerning the "management" topic, no peculiar problems are forecasted in terms of safety and risk management. To ensure the access to the multidisciplinary service, a tight coordination with territorial specialists will be necessary. This is of high importance, considering the aforementioned role of general practitioners, dermatologists and rheumatologists operating within territorial health centers, whom will address the patients on the base of the symptoms assessed during the visits.

No critical points are envisaged in cultural terms in the acceptance of the multidisciplinary service by patients, for whom an increase in the satisfaction for the services delivered might be observed due to the reduced time to diagnosis. The personnel of each hospital in which the service will be activated should not have any resistance towards its implementation, not being related to an increase of daily workload. In terms of interest groups to be taken into consideration for the implementation of the technology, they are those already considered in the communication activity described above.

\section{Discussion and conclusions}

The optimized pathway for the diagnosis and management of psoriatic arthritis proposed would have a limited organizational impact at both hospital and Regional Health Service levels, while leading to theoretical benefits in terms of a prompt diagnosis of the pathology.

Furthermore, the implementation of the multidisciplinary service is in line with the objectives of the resolutions of the Regional Council of Lombardy Region for the "re-organization of the management pathway of patients affected with chronic pathologies" [16,17]. The service would, in fact, allow the performance of an empowerment activity on patients, concerning their therapeutic and monitoring pathway, giving the possibility to structure an individual care plan shared by the two specialists involved (dermatologist and rheumatologist), optimizing the process.

In terms of health care marketing, the implementation of the service would have a positive impact on patients' experience, improving the quality of the service provided, thanks to the multidisciplinary approach adopted, limiting the patients' resources needed for the diagnosis, reducing the number of visits and time loss. The optimized pathway, therefore, would have a limited impact on the marketing mix (in terms of typology of service provided, costs for patients, setting, and communication activities), while potentially improving patients satisfaction, increasing the possibility of patients' retention.

The main limit of the analysis conducted is related to its "monocentric" approach and this might limit the generalizability of the results. The analysis presented should be considered as a preliminary step for a further wider analysis, considering the involvement of different stakeholders, as patients, general practitioners, hospital managers, and increasing the number of dermatologists and rheumatologists involved.

To successfully implement the dermatological and rheumatologic multidisciplinary service, a precise communication strategy is mandatory, as explained above.

At a Regional level, training activities addressed to general practitioners should also be promoted to increase their capacity to recognize symptoms that might be related to psoriatic arthritis, reducing the time to referral to the multidisciplinary service. 


\section{Acknowledgement}

The HTA Core Model ${ }^{\circledR}$, developed within EUnetHTA (www.eunethta.eu), has been utilized when producing the contents and/or structure of this work. The following version of the Model was used: HTA Core Model ${ }^{\circledR}$ Version 3.0. Use of the HTA Core Model does not guarantee the accuracy, completeness, quality or usefulness of any information or service produced or provided by using the Model.

\section{Funding}

The analysis was performed thanks to an unconditional grant from Novartis Italia.

\section{Conflicts of interest}

- UR reports grants from Novartis Italia, during the conduct of the study;personal fees from Astrazeneca, Bayer, Italfarmaco, outside the submitted work.

- CS reports consulting/speakers fee from AbbVie, Aesku, Alfa-Wassermann, BMS, Biogen, Celgene, Eli-Lilly, Grifols, Janssen, MSD, Novartis, Pfizer, Roche, Sanofi-Genzyme, UCB; research support from AbbVie, Janssen, MSD, Novartis, Pfizer; travel support from Celgene, Eli-Lilly, Grunenthal, Janssen, Novartis, Pfizer, Roche, UCB, outside the submitted work.

- VP reports grants from Novartis Italia, during the conduct of the study.

- DC reports grants from Novartis Italia, during the conduct of the study; personal fees from Mundipharma, Takeda, Abbvie, outside the submitted work.

- AC reports consulting/speakers fee or grants for research from Novartis, Janssen, Abbvie, UCB, Lilly, Leo Pharma, UCB, outside the submitted work.

\section{REFERENCE}

1. Bowcock AM, Cookson WO. The genetics of psoriasis, psoriatic arthritis and atopic dermatitis. Hum Mol Genet 2004; 13: R43-55; https://doi.org/10.1093/hmg/ddh094

2. de Vlam K, Lories RJ. Remission in psoriatic arthritis. Curr Rheumatol Rep 2008; 10 : 297-302; https://doi.org/10.1007/s11926-008-0048-Z

3. Dhir V, Aggarwal A. Psoriatic arthritis: a critical review. Clin Rev Allergy Immunol 2013; 44: 141-8; https://doi.org/10.1007/s12016-012-8302-6

4. Mortezavi M, Thiele R, Ritchlin C. The joint in psoriatic arthritis. Clin Exp Rheumatol 2015; 33: S20-5

5. Veale DJ, Fearon U. The pathogenesis of psoriatic arthritis. Lancet 2018; 391: 2273-84; https://doi.org/10.1016/S0140-6736(18)30830-4

6. Scotti L, Franchi M, Marchesoni A, et al. Prevalence and incidence of psoriatic arthritis: A systematic review and meta-analysis. Semin Arthritis Rheum 2018; 48: 28-34; https://doi. org/10.1016/j.semarthrit.2018.01.003

7. Prignano F, Rogai V, Cavallucci E et al. Epidemiology of Psoriasis and Psoriatic Arthritis in Italy-a Systematic Review. Curr Rheumatol Rep 2018; 20: 43; https://doi.org/10.1007/ s11926-018-0753-1

8. Hellgren L. Association between rheumatoid arthritis and psoriasis in total populations. Acta Rheumatol Scand 1969; 15: 316-26; https://doi.org/10.3109/rhe1.1969.15.issue-1-4.40

9. Hoff M, Gulati AM, Romundstad PR, et al. Prevalence and incidence rates of psoriatic arthritis in central Norway: data from the Nord Trøndelag health study (HUNT). Ann Rheum Dis 2015; 74: 60-4; https://doi.org/10.1136/annrheumdis-2013-203862

10. Salaffi F, De Angelis R, Grassi W; MArche Pain Prevalence; INvestigation Group (MAPPING) study. Prevalence of musculoskeletal conditions in an Italian population sample: results of a regional community-based study. I. The MAPPING study. Clin Exp Rheumatol 2005; 23: 819-28

11. De Angelis R, Salaffi F, Grassi W. Prevalence of spondyloarthropathies in an Italian population sample: a regional community-based study. Scand J Rheumatol 2007; 36: 14-21; https://doi.org/10.1080/03009740600904243

12. Cimmino MA, Zampogna A, Murroni S, et al. Methodology of an epidemiologic prevalence study in rheumatology: the Chiavari study. Reumatismo 2002; 54: 40-7; https://doi. org/10.4081/reumatismo.2002.40 
13. EUnetHTA Joint Action 2, Work Package 8. HTA Core Model ${ }^{\circledR}$ version 3.0 (Pdf); 2016. Available at www.htacoremodel.info/BrowseModel.aspx (last accessed November 2018)

14. Souza CS, de Castro CCS, Carneiro FRO, et al. Metabolic syndrome and psoriatic arthritis among patients with psoriasis vulgaris: Quality of life and prevalence. J Dermatol 2019; 46: 3-10; https://doi.org/10.1111/1346-8138.14706

15. Truong B, Rich-Garg N, Ehst BD, et al. Demographics, clinical disease characteristics, and quality of life in a large cohort of psoriasis patients with and without psoriatic arthritis. Clin Cosmet Investig Dermatol 2015; 8: 563-9; https://doi.org/10.2147/CCID.S90270

16. Regional Council of Lombardy Region. Resolution X/6164 - 30/01/2017. “Governo della domanda: avvio della presa in carico di pazienti cronici e fragili. Determinazioni in attuazione dell'art. 9 della legge n. 23/2015". Available at http://www.regione.lombardia.it/ (last accessed November 2018)

17. Regional Council of Lombardy Region. Resolution X/6551 - 04/05/2017. "Riordino della rete d'offerta e modalità di presa in carico dei pazienti cronici e/o fragili in attuazione dell'art.9 della legge regionale n.33/2009". Available at Available at http://www.regione. lombardia.it/ (last accessed November 2018) 